Recibido:

25-XI-2019

Aceptado:

5-XII-2019

Publicado en línea:

19-XII-2019
Dimorfismo sexual a través del análisis morfométrico de bóvedas palatinas en una población peruana

\section{Sexual Dimorphism Via Palatal Vault Morphometric Analysis on A} Sample Peruvian Population

Karen J. Escalante-Flórez DDS1; Daniel G. Suárez-Ponce DDS, MSc, PhD²; Ymelda W. Velezmoro-Montes DDS, MSc ${ }^{3}$

1. Odontólogo. Universidad Antonio Nariño, Cúcuta-Colombia. Odontóloga Forense. Universidad Científica del Sur, Lima-Perú.

2. Docente Departamento de Estomatología Médico Quirúrgico. Facultad de Odontología. Universidad Nacional Mayor de San Marcos, Perú.

3. Docente Coordinadora de la Especialidad en Odontología Forense. Universidad Científica del Sur, Perú.

Autor para correspondencia: Dra. Karen Julieth Escalante Flórez - karenescalanteflorez@gmail.com

RESUMEN: Objetivo: Determinar la precisión del dimorfismo sexual a través del análisis morfométrico de bóvedas palatinas en cráneos procedentes del Laboratorio de Investigación Forense del Equipo Forense Especializado (EFE) de Ayacucho del Ministerio Público - Perú. Materiales y métodos: Diseño transversal, constituido con una muestra de 43 cráneos con sexo biológico preestablecido, (24=masculino y $19=$ femenino). La estimación del dimorfismo sexual se estableció a través del método propuesto por Meera Jacob y cols., basado en las mediciones de la longitud y ancho palatino, posteriormente se calculó el índice palatino, estableciéndose la precisión y exactitud del método. El análisis inferencial se realizó con un nivel de significancia del 5\% a través de la prueba T student, Shapiro Wilk y el coeficiente de Pearson. Resultados: Se estableció que el ancho palatino presenta una media de $3,43(+0,31)$ $\mathrm{cm}$. para el sexo masculino y $3,62(+0,25) \mathrm{cm}$. para el femenino, en la longitud palatina la media fue de $5,07(+0,44) \mathrm{cm}$. para masculino y $4,79(+0,41) \mathrm{cm}$. en el femenino; en el índice palatino, el $100 \%$ de los cráneos de sexo masculino corresponden al paladar duro estrecho y en el femenino un 73,7\% al estrecho, un $21 \%$ al intermedio y un $5,3 \%$ al ancho. Además, se determinó que existió diferencias estadísticamente significativas entre los parámetros del ancho y longitud de la bóveda palatina entre ambos sexos y se planteó una fórmula de análisis discriminante. Conclusión: El análisis morfométrico de bóvedas palatinas establece una precisión de 83.72\% en la estimación del dimorfismo sexual.

PALABRAS CLAVES: Dimorfismo sexual; Bóvedas palatinas; Análisis morfométrico.

ESCALANTE K., SUÁREZ D., VELEZMORO Y., 2020: Dimorfismo sexual a través del análisis morfométrico de bóvedas palatinas en una población peruana.-ODOVTOS-Int. J. Dental Sc., 22-2 (May-August): 133-141. 
ABSTRACTS: Objective: Determine sexual dimorphism precision via palatal vault morphometric analysis in craniums sourced from the Laboratorio de Investigación Forense del Equipo Forense Especializado (EFE) de Ayacucho del Ministerio Público - Perú. Materials and Methods: Cross-sectional design consisting of a sample of 43 skulls with pre-established biological gender, (24 males and 19 females). Sexual dimorphism was established by means of the method proposed by Meera Jacob and co., based on palatal width and length measurements, after which the palatal index was calculated, establishing the method's precision and accuracy. The inferential analysis was made with a level of significance of $5 \%$ through the $T$ student test, Shapiro Wilk and Pearson's coefficient. Results: Palatal width had a mean of 3.43 $(+0.31 \mathrm{~cm})$ for males and $3.62(+0.25)$ for females and in palatal length a mean of $5.07(+0.44) \mathrm{cm}$. for males and $4.79(+0.41) \mathrm{cm}$. for females is established; on palatal index, $100 \%$ of the male skulls correspond to hard narrow palate and on females $73.7 \%$ to narrow, $21 \%$ to intermediate and $5.3 \%$ to wide. Furthermore, it was determined that there were statistically significant differences between width and length of palatal vault for both genders, and a discriminating analysis formula was proposed. Conclusion: Morphometric analyses of palatal vaults establish an $83.72 \%$ precision on the estimation of sexual dimorphism.

KEYWORDS: Sexual dimorphism; Palatal vaults; Morphometric analysis.

\section{INTRODUCCIÓN}

El propósito de la identificación forense reside en establecer la individualidad del sujeto; (1) requiriendo de un grupo de especialidades con conocimientos de las diferentes ramas aplicadas a los aspectos legales y de carácter forense como lo son: la criminalística, la antropología forense, la odontología forense, la medicina legal y el derecho; que trabajan de manera múltiple e interdisciplinaria, encaminadas a la solución de problemas que auxilian a la justicia (1-2).

En las ciencias forenses un aspecto importante es establecer la identidad, de aquellos individuos que lo han perdido por diversas circunstancias, a través de la agrupación de caracteres que individualizan a una persona y la diferencia de los demás, permitiendo la identificación basada en la recolección y asociación sistemática de los caracteres individualizantes (3). La necesidad de identificar restos humanos (cadáveres en estado de descomposición, desmembramiento, mutilación y restos óseos) en odontología forense aparecen naturalmente como resultado de los grandes desastres naturales y no naturales que han afectado al hombre desde los albores de la humanidad (4).

La odontología forense es una especialidad de vital importancia en la identificación humana que aplica sus conocimientos (clínicos, prácticos y teóricos) de esta ciencia-arte para desarrollar el correcto examen, manejo, valoración y presentación de evidencia bucodentomaxilar y craneofacial en disposición de la norma jurídica (2). El odontólogo forense en el estudio de restos humanos esqueletizados se encargará de establecer el big four (gran cuatro) que consiste en estimar el sexo, edad, estatura y patrón ancestral para hallar el perfil biológico de un individuo; (2) aplicando diversas metodologías basadas en aspectos cualitativos y cuantitativos con el fin de reconstruir parámetros biológicos, traumáticos y / o tafonómicos (5). 
El dimorfismo sexual corresponde a las características corporales que distingue los hombres de las mujeres, mediante el tamaño de sus estructuras; que por lo general en los hombres son más grandes, robustas; y en mujeres más pequeñas y gráciles (6).

La estimacion del sexo es una parte fundamental de la reconstrucción del perfil biológico confiable al examinar restos óseos, (7) siguiendo patrones establecidos. William y Rogers (2006) manifiestan que con el cráneo se puede establecer de 80 a $90 \%$ la certeza de sexo y Krogman e Iscan (1986) reportan que la pelvis permite obtener una confiabilidad de precisión de 90 a 95\% (8). El cráneo se considera la segunda estructura ósea más confiable para estimar el sexo de una persona adulta (9). En la estimación del sexo a través del estudio del cráneo, se toman en cuenta parámetros de análisis morfológico y métrico de los caracteres bucodentomaxilares, como la forma $y / 0$ profundidad del paladar y el alineamiento dental. El paladar consta de dos porciones, el duro y el blando. El paladar duro, o bóveda palatina, es de estructura ósea y es el más anterior; (10) contribuye a la separación de las cavidades oral y nasal; (11) está constituida por los procesos palatinos del maxilar superior y las placas horizontales de los huesos palatinos posteriores (12). El paladar duro es altamente resistente y se encuentra aislado en la base del cráneo, es por ello que a menudo está disponible para el análisis forense, incluso cuando otros componentes esqueléticos superficiales están deteriorados, (4) así mismo, establece diferencias entre el sexo, en cuanto al paladar masculino desde el análisis morfológico es por lo general ancho y profundo pero en el sexo femenino tiende a ser profundo y estrecho, así mismo, el arco dentario masculino es grueso y en el femenino sutil, los bordes alveolares son más verticales en el sexo masculino que en el femenino (2). La importancia de la odontología forense en la identificación humana se ve corroborado por el hecho de que las estructuras palatinas resisten la descomposición postmortem durante varios años y más en los tejidos dentales (13). Según Sumati y cols. (14) 2012, revelaron que el tamaño de paladar clasificó correctamente el sexo con una precisión de 70\% y encontraron que es el mejor factor determinante de sexo. Jacob y cols. (15) 2016 concluyeron que la longitud máxima del paladar y la anchura es mayor en los hombres que en las mujeres y que según el índice palatino, la mayoría de los paladares duros pertenecían a la categoría de tipo ancho en una población del sur de la India. Kamath y cols. (16) 2016, observaron que la longitud del paladar es un marcador sexual definitivo con una previsibilidad sexual del $87,2 \%$. Kumar y cols. (17) 2016, mostraron diferencias muy significativas de la longitud y la anchura del paladar entre ambos sexos. El índice palatino mostró que la mayoría de los cráneos tenían un paladar estrecho. Alves y cols. (18) 2018, demostraron que si existe dimorfismo sexual en el análisis métrico y no métrico del paladar duro. Mustafa y cols. (19) 2019, mediante los tres predictores (Iongitud, ancho y profundidad) indicaron que estaban significativamente correlacionados con el género en el grupo de adultos y establecieron medidas mayores en hombres que en mujeres.

En la presente investigación se analizaron las medidas morfométricas del paladar duro orientadas a la estimación del dimorfismo sexual, siendo estas: 1) Longitud palatina: distancia entre el orale anterior (punto en el extremo anterior de la sutura incisiva ubicada entre las cuencas de los dos incisivos centrales superiores) hacia la espina nasal posterior en la parte posterior. 2) Anchura palatina: distancia entre los bordes internos de las cavidades de los segundos molares superiores (endomolare). 3) Índice palatino: se calculó mediante la fórmula: Ancho palatino / Longitud palatina x 100; (15) este estudio es importante porque permitirá que los odontólogos forenses trabajen de manera multidisciplinaria con los diversos especialistas de las ciencias forenses, 
empleando un nuevo método que ayudara a reconstruir el perfil biológico de los restos óseos.

El propósito del estudio fue determinar la precisión del dimorfismo sexual a través del análisis morfométrico de bóvedas palatinas en cráneos procedentes del Laboratorio de Investigación Forense del Equipo Forense Especializado (EFE) de Ayacucho - Perú.

\section{MATERIALES Y MÉTODOS}

El estudio fue de tipo descriptivo, comparativo, observacional y transversal, realizándose mediciones en un total de 43 cráneos; 19 pertenecían al sexo femenino y 24 al sexo masculino. La investigación fue aprobada por el Comité Institucional de Ética en Investigación de la Universidad Científica del Sur, así mismo, fue aprobada y autorizada por la Jefatura Nacional del Instituto de Medicina Legal y Ciencias Forense del Perú.

Las muestras fueron obtenidas del Laboratorio de Investigación Forense del Equipo Forense Especializado (EFE) de Ayacucho - Perú; teniendo en cuenta los criterios de inclusión cráneos completos, con bóvedas palatinas en buen estado de conservación, cráneos con sexo establecido, cráneos con los segundos molares superiores 0 con su proceso alveolar sin reabsorción y cráneos que corresponden a sujetos adultos; se excluyeron cráneos edéntulos parcial con reabsorción completa del proceso alveolar a nivel de los segundos molares superiores y bóvedas palatinas con patologías y/o malformaciones.

Se realizó la calibración con un especialista en Odontología Forense, obteniéndose en la calibración interobservador para la medición de la longitud del paladar duro, un valor de 0,88 y para el ancho del paladar duro de 0,96; y para la calibración intraobservador en la primera medición un resultado de 0,88 y en una segunda medición de 0,94, expresando una fiabilidad excelente.
Las mediciones las bóvedas palatinas se tomaron de acuerdo al autor Meera Jacob y colaboradores (15), con un calibrador Vernier digital determinándose la longitud palatina (distancia entre el punto más anterior de la fosa incisiva hasta la espina nasal posterior) (Figura 1) y el ancho palatino (distancia entre los bordes internos de las cavidades de los segundos molares superiores) (Figura 2) y el índice palatino se calculó mediante la fórmula Ancho palatino / Longitud palatina x 100 .

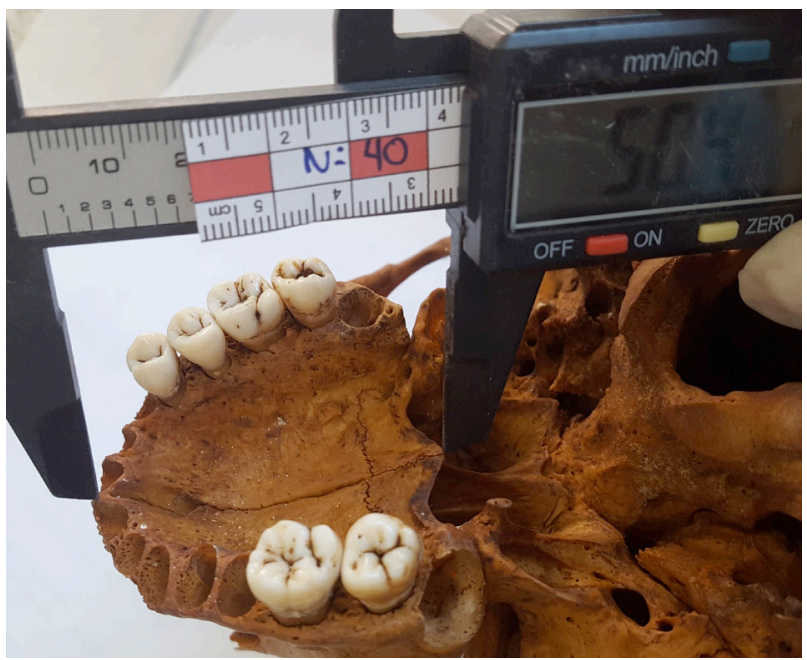

Figura 1. Medición del diámetro anteroposterior (Iongitud) de la bóveda palatina.

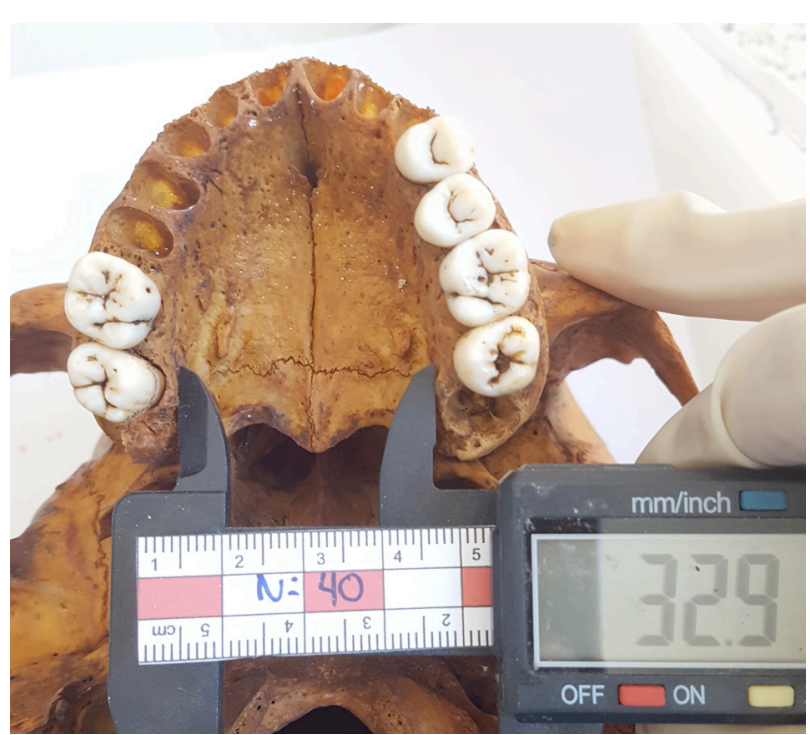

Figura 2. Medición del diámetro transversal (ancho) de la bóveda palatina 
Para el procesamiento de datos se empleó el programa IBM SPSS versión 25, realizándose el análisis descriptivo y la frecuencia de la distribución de la muestra según sexo; se estableció el mínimo, máximo, media y desviación estándar del ancho, longitud e índice palatino según sexo. Además, se obtuvieron las pruebas de normalidad de variables (paramétricas) donde expresaron que, si hay normalidad, por obtener valores $>0,05$; se aplicó la prueba de Shapiro-Wilk para el análisis de las 43 muestras. Habiendo normalidad de la prueba paramétrica, se prosiguió a realizar la prueba de t student, la cual estableció como resultado valores estadísticamente significativos entre el sexo masculino y femenino $(p<0.05)$. Posteriormente, se aplicó la prueba de correlación de bivariables (Pearson) siendo el nivel de significancia $<0,050$ expresando la existencia de relación significativa entre las variables (ancho y longitud palatina) y el sexo. Finalmente se realizó un análisis discriminante utilizando mediciones para discriminar entre ambos sexos (Wilks Lambda).

\section{RESULTADOS}

Se observa en la Tabla 1, que la longitud palatina en el sexo masculino es de 5,07 ( \pm $0,44) \mathrm{cm}$. y para el femenino es de 4,79 $( \pm$ $0,41) \mathrm{cm}$.

En la tabla 2, se observa que la media para el ancho palatino en el sexo masculino es de $3,43( \pm 0,31) \mathrm{cm}$. y en el femenino es de 3,62 ( \pm $0,25) \mathrm{cm}$.

El tipo de paladar y su relación con el sexo se presenta en la Tabla 3.

En la Tabla 4 se presenta el análisis para discriminar el dimorfismo sexual.

En la Tabla 5 se observa que la precisión de dimorfismo sexual según la longitud palatina es de $65,11 \%$ y el ancho palatino de $62,79 \%$ y para ambos es de $83.72 \%$.

Tabla 1. Dimorfismo sexual mediante la longitud de las bóvedas palatinas.

\begin{tabular}{cccccc}
\hline Sexo & $\begin{array}{c}\text { Morfometría } \\
\text { del paladar }\end{array}$ & Mínimo & Máximo & Media & $\begin{array}{c}\text { Desviación } \\
\text { estándar }\end{array}$ \\
\hline Masculino & $\begin{array}{c}\text { Longitud palatina } \\
(\mathrm{cm})\end{array}$ & 4,22 & 5,95 & 5,0788 &, 44453 \\
Femenino & & 4,10 & 5,52 & 4,7958 &, 41444 \\
\hline
\end{tabular}

Tabla 2. Dimorfismo sexual mediante el ancho de las bóvedas palatinas.

\begin{tabular}{cccccc}
\hline Sexo & $\begin{array}{c}\text { Morfometría } \\
\text { del paladar }\end{array}$ & Mínimo & Máximo & Media & $\begin{array}{c}\text { Desviación } \\
\text { estándar }\end{array}$ \\
\hline Masculino & Ancho palatino $(\mathrm{cm})$ & 2,82 & 4,04 & 3,4329 &, 31281 \\
Femenino & & 3,02 & 4,05 & 3,6268 &, 25434 \\
\hline
\end{tabular}

Tabla 3. Tipo de paladar duro según sexo.

\begin{tabular}{cccccc}
\hline Sexo & Mínimo & Máximo & Media & $\begin{array}{c}\text { Desviación } \\
\text { estándar }\end{array}$ & Tipo de paladar duro \\
\hline Masculino & 58,36 & 75,16 & 67,72 & 4,60 & $100 \%$ paladar duro estrecho \\
Femenino & 66,17 & 89,88 & 75,93 & 6,11 & $73,7 \%$ paladar estrecho, \\
& & & & $21,0 \%$ intermedio y \\
& & & & & $5,3 \%$ ancho. \\
\hline
\end{tabular}


Tabla 4. Análisis discriminante utilizando mediciones para discriminar entre sexos.

\begin{tabular}{|c|c|c|c|}
\hline \multicolumn{4}{|l|}{$\begin{array}{l}\text { Ancho palatino } \\
D=-12.192+3.465 \text { (ancho palatino) }\end{array}$} \\
\hline Wilks Lambda $=0.895, p$-value $=0.034<0.050$ & Femenino & Masculino & General \\
\hline Porcentaje de grupo con una predicción precisa & $73.7 \%$ & $54.2 \%$ & $62.79 \%$ \\
\hline Función centroide de grupo & 0.375 & -0.297 & $\begin{array}{l}\text { Varones } \\
D<-0.200\end{array}$ \\
\hline \multicolumn{4}{|l|}{$\begin{array}{l}\text { Longitud palatina } \\
D=-11.478+2.317 \text { (longitud palatina) }\end{array}$} \\
\hline Wilks Lambda $=0.900, p$-value $=0.039<0.050$ & Femenino & Masculino & General \\
\hline Porcentaje de grupo con una predicción precisa & $63.2 \%$ & $66.7 \%$ & $65.11 \%$ \\
\hline Función centroide de grupo & -0.366 & 0.290 & $\begin{array}{l}\text { Varones } \\
D>0.01\end{array}$ \\
\hline \multicolumn{4}{|c|}{$\begin{array}{l}D=-0.915-2.718 \text { (longitud palatina) }+4.087 \text { (ancho } \\
\text { palatino) }\end{array}$} \\
\hline Wilks Lambda $=0.613, p$-value $=0.000<0.050$ & Femenino & Masculino & General \\
\hline Porcentaje de grupo con una predicción precisa & $84.2 \%$ & $83.3 \%$ & $83.72 \%$ \\
\hline Función centroide de grupo & 0.872 & -0.690 & $\begin{array}{l}\text { Varones } \\
D<-0.1\end{array}$ \\
\hline
\end{tabular}

Tabla 5. Precisión del dimorfismo sexual a través del análisis morfométrico de la bóveda palatina.

\begin{tabular}{cccc}
\hline $\begin{array}{c}\text { Morfometría } \\
\text { del paladar duro }\end{array}$ & Masculino & Femenino & \% General predictivo \\
\hline Ancho palatino & $54.2 \%$ & $73.7 \%$ & $62.79 \%$ \\
Longitud palatina & $66.7 \%$ & $63.2 \%$ & $65.11 \%$ \\
Longitud palatina + Ancho palatino & $84.2 \%$ & $83.3 \%$ & $83.72 \%$ \\
\hline
\end{tabular}

\section{DISCUSIÓN}

El objetivo de la presente investigación fue determinar la precisión del dimorfismo sexual a través del análisis morfométrico de bóvedas palatinas en cráneos procedentes del Laboratorio de Investigación Forense del Equipo Forense Especializado (EFE) de Ayacucho del Ministerio Público - Perú. Los resultados establecen una alta precisión entre el dimorfismo sexual y el análisis morfométrico de bóvedas palatinas.

Además, se obtuvo que la longitud palatina tiene mayor precisión para estimar el dimorfismo sexual en comparación con el ancho de las bóvedas palatinas, en el análisis la longitud palatina establece un dimorfismo sexual mayor en el masculino que en el femenino y el ancho palatino establece un dimorfismo sexual mayor en el sexo femenino que en el sexo masculino. Se calculó el índice palatino según sexo, determinándose que en el sexo masculino predominó el paladar duro estrecho y en el sexo femenino el paladar duro estrecho, seguido del paladar duro intermedio.

En la presente investigación los resultados obtenidos para el ancho palatino fueron comparados con los evidenciados por otros autores, encontrando 
coincidencias de los promedios correspondientes al sexo masculino $(3.43 \mathrm{~cm})$ con: Meera Jacob y cols. (15) en su estudio de 90 cráneos en la región del Sur de la India, quienes obtuvieron un valor de media de $3.53 \mathrm{~cm}$; Makhani y cols. (20) quienes estudiaron 108 cráneos de la India y revelaron un valor de $35.31 \mathrm{~mm}$. En cuanto al sexo femenino $(3.63 \mathrm{~cm})$, Saadia y cols. (21) tomaron muestras de 100 cráneos egipcios consiguiendo un valor de media de $37.71 \mathrm{~mm}$.

De modo similar, los valores de los autores que más se acercaron a los resultados de la longitud palatina, para el sexo masculino $(5.07 \mathrm{~cm})$, fueron: Anil Kumar y cols. (17) en su estudio de 86 cráneos en Delhi-India, quienes alcanzaron valores promedio de $52.5 \mathrm{~mm}$; Saadia y cols. (21) hallaron un valor de $52.77 \mathrm{~mm}$. Para el sexo femenino $(4.79 \mathrm{~cm})$, Abilasha y cols. (22) en 100 cráneos de la región central de la India, mostraron valores de $46.06 \mathrm{~mm}$.

Aproximadamente todos los valores de la longitud y el ancho del paladar, manifestados por los autores mencionados, fueron mayores en el sexo masculino que en el femenino, a excepción de Vabushana y cols. (23) quienes, en una muestra de 40 cráneos en Chennai, Tamil Nadu-India; lograron valores mayores en el sexo femenino $(32.06 \mathrm{~mm})$ que en el sexo masculino $(30.34 \mathrm{~mm})$ para el ancho palatino, dando concordancia con los resultados de la presente investigación y siendo el valor más resaltante.
En este estudio, el tipo de paladar duro que predominó fue el estrecho tanto en el sexo masculino como en el femenino; al igual que en Ios estudios de los autores Meera Jacob y cols. (15) en el sexo femenino, Anil Kumar y cols. (17) y Abilasha y cols. (22) en ambos sexos, Orish e Ibeachu (24) en 100 cráneos de Nigeria predominó el paladar estrecho en el sexo masculino y Saadia y cols. (21) en ambos sexos. Es decir que los estudios realizados en poblaciones de la India, Nigeria y Egipto, prevaleció el mismo tipo de paladar duro que en la población peruana de Ayacucho; sin embargo, los resultados no se pueden generalizar porque son aplicables para cada una de las poblaciones estudiadas.

Otro aspecto importante a sobresalir es que el porcentaje de la precisión del sexo mediante el análisis morfométrico de las bóvedas palatinas fue de $83.72 \%$, encontrándose dentro del rango (80 a $90 \%$ ) que los autores William y Rogers (2006) establecen de confiabilidad para la certeza del sexo con el cráneo (8).

\section{CONCLUSIÓN}

El análisis morfométrico de bóvedas palatinas permite establecer una precisión de $83.72 \%$ en la estimación del dimorfismo sexual en cráneos procedentes del Laboratorio de Investigación Forense del Equipo Forense Especializado (EFE) de Ayacucho del Ministerio Público - Perú. 


\section{REFERENCIAS}

1. Lozano y Andrade O. Estomatología forense. México Trillas, 2006.

2. Correa R. A. Odontología forense. 2da ed. México: Trillas, 2011.

3. Correa R. A. Identificación forense. México: Trillas, 1990.

4. Krishan K., Chatterjee P. M., Kanchan T., et al. A review of sex estimation techniques during examination of skeletal remains in forensic anthropology casework. Forensic Sci Int. 2016; 261: 165.e1-165.e8, doi: 10.1016/j.forsciint. 2016.02.007, indexed in Pubmed: 26926105.

5. Krenzer U. Compendio de métodos antropológico forenses para la reconstrucción del perfil osteo-biológico. Tomo 1 Osteometría. $1^{a}$ ed. Guatemala: Cafca; 2006.

6. Gonzáles C. G., Rojas M. P., Marroquín T. Y. Odontología forense y la reconstrucción del perfil biológico humano: sexo, ancestro, edad biológica y estatura. Odontología forense: identificación humana y alteraciones del sistema estomatognático en el contexto forense. Bogotá D.C., Colombia. 2018; 205-243.

7. Steadman D. W., Haglund W. D. The scope of anthropological contributions to human rights investigations. J Forensic Sci. 2005; 50 (1): 23-30, indexed in Pubmed: 15830993.

8. Osorio R. H., Sanabria C. Dimorfismo sexual en tejidos óseos. Patología y antropología forense de la muerte: la investigación científico-judicial de la muerte y la tortura, desde las fosas clandestinas, hasta la audiencia pública. Bogotá D.C., Colombia: Forensic Publisher. 2016; 179-220.
9. Best KC, Garvin HM, Cabo LL. An Investigation into the Relationship between Human Cranial and Pelvic Sexual Dimorphism. J Forensic Sci. 2018; 63 (4): 990-1000, doi: 10.1111/1556-4029.13669, indexed in Pubmed: 29044526.

10. Revuelta R. La cavidad oral del nacimiento a la infancia: desarrollo, patologías y cuidados. Perinatol Reprod Hum. 2009; 23 (2): 82-89.

11. Tangugsorn V., Skatvedt O., Krogstad O., Lyberg T. Obstructive sleep apnoea: a cephalometric study. Part-I. Cervicocraniofacial skeletal morphology. Eur J Orthod. 1995; 17 (1): 45-6.

12. Varakshmi K. L., Sangeeta M., Shilpa N., Arunashri A. An osteological study of morphometry of hard palate and its importance. International Journal of Research in medical sciences. 2015; 3 (9): 2210-2213.

13. Caldas I. M., Magalhaes T., Afonso A. Establishing identity using cheiloscopy and palatoscopy. Forensic Science International. 2007; 165 (1): 1-9.

14. Sumati, Patnaik V., Phatak A. Determination of sex from hard palate by discriminant function analysis. International Journal of Basic and Applied Medical Science. 2012; 2 (3): 243-251.

15. Jacob M., Bindhu S., Avadhani R. Sex determination from hard palate measurements using palatine index with reference to its clinical implications. Indian Journal of Clinical Anatomy and Physiology. 2016; 3 (2); 186-188.

16. Kamath V., Asif M., Shetty R. Avadhani R. Binary logistic regression analysis of hard palate dimensions for sexing human crania. Anat Cell Biol. 2016; 49 (2): 151-159. 
17. Kumar A., Ajmani M. L., Heming T. Morphological and morphometric study of hard palate in Indian population. International Journal of Biomedical Research. 2016; 7 (11): 778-784.

18. Alves N., Deane N. F., Ceballos F., Hernández P., Gonzáles J. Sex prediction by metric and non-metrci analysis of the hard palate and the pyriform apertura. Folia Morphol. Jun 2018; 78 (1): 137-144.

19. Mustafa A. G., Tashtoush A. A., Alshboul O. A., Allouh M. Z., Altarifi A. A. Morphometric Study of the Hard Palate and Its Relevance to Dental and Forensic Sciences. International Journal of Dentistry. 2019. ID: 1687345.

20. Petkar M. R., Makhani C. S., Datir S. B., Farooqui J., Bangal R. S., Chavan K. D. Sexing of skull by metric analysis of hard palate. International Journal of Medical Toxicology Legal Medicine. 2017, 20 (3-4): 52-54.

21. Saadia A. Shalaby, Esam M. Eid, Naglaa A.S. Sarg, Amany M.A. Sewilam. Morphometric analysis of hard palate in Egyptian skulls. Benha Medical Journal. 2015, 32: 59-72.

22. Abilasha M. W., Rajeshere A. N. A study of palatal indices and foramina in the hard palate of adult human skulls in central india región. Int J Anat Res. 2019, 7 (2.1): 6397-03.

23. Vabushana V. J., Thenmozhi M. S., Lakshmanan G. Determination of sex using various parameters of hard palate. Drug Invention Today. 2019, 12: 1018-1020.

24. Orish C. N., Ibeachu P. C. Craniometric indices of Nigeria skulls. Int J Anat Appl Physiol. 2016, 2 (1), 6-13. 